\title{
High-affinity T cell receptors for adoptive cell transfer
}

\author{
Ariel Isser ${ }^{1}$ and Jonathan P. Schneck $k^{2,3}$ \\ 'Department of Biomedical Engineering, 'Department of Pathology, Medicine and Oncology, and ${ }^{3}$ Immunology Program, Institute of Cellular Engineering, Johns Hopkins University, School of Medicine, \\ Baltimore, Maryland, USA.
}

\begin{abstract}
Adoptive cell transfer (ACT) of engineered T cell receptors (TCRs) for cancer immunotherapy has evolved from simple gene transfer of isolated TCRs to various affinity enhancement techniques that overcome limitations imposed by central and peripheral tolerance on TCR affinity. In the current issue of the $J C I$, Poncette et al. used mice with human TCR $\alpha \beta$ and HLA gene loci to discover CD4+ TCRs of optimal affinity for cancer testis antigen (CTA) NY-ESO-1. They combined this TCR with a previously discovered NY-ESO-1specific CD8+ TCR in an ACT fibrosarcoma tumor model to demonstrate the importance of $\mathrm{T}$ cell help in mediating antitumor responses.
\end{abstract}

\section{Evolution of T cell receptor engineering}

The first demonstration that $\mathrm{T}$ cells could be engineered with a predetermined specificity through transfer of the $\alpha$ and $\beta$ T cell receptor (TCR) genes occurred more than 30 years ago (1). Two decades later, this technology was applied clinically for the first time in adoptive cell transfer (ACT) treatment of metastatic melanoma using MART-1 melanoma antigen-specific clones recovered from tumor-infiltrating lymphocytes (TILs) (2). While in the initial trials 2 of 15 patients (13\%) had objective tumor regression after treatment, subsequent trials targeting MART- 1 and gp100 melanoma antigens with high-avidity TCRs (based on IFN- $\gamma$ secretions with titrating amounts of cognate antigen) yielded higher response rates (30\% and $20 \%$, respectively). However, this treatment led to widespread destruction of healthy melanocytes and significant on-target toxicities (3). Nevertheless, as the high-avidity gp100 antigen was discovered by immunizing HLA-A2transgenic mice with the ${\text { gp } 10 O_{154-162}}$ epitope, this study demonstrated for the first time the clinical potential of tumor antigen-specific TCRs generated in nonhuman hosts whose $\mathrm{T}$ cell responses to human antigens are unencumbered by limitations posed by central or peripheral tolerance (3). More recently, a murine TCR (mTCR) gene specific to the cancer testis antigen (CTA) New York esophageal squamous cell carcinoma 1 (NY-ESO-1) was isolated from HLA-A2-transgenic mice immunized with NY-ESO- ${ }_{157-165}$ peptide and is being used in an ongoing phase II clinical trial (NCT01967823) (4). Other groups have used alloreactive settings (5), rational design through mutations in the complementarity-determining regions (CDRs) of TCRs (6), yeast (7), phage display (8), or some combination thereof to overcome tolerance-imposed limitations on tumor-associated antigen (TAA) TCR avidity.

\section{Engineering "optimal”-affinity \\ T cell receptors using a nontolerant host}

In the current issue of the JCI, Poncette and colleagues use a system that has been developed over several years by the Blankenstein laboratory to generate "optimal"affinity TCRs that bind human HLA, but recognize self-antigens such as MART-1 and NY-ESO-1 as foreign (9). Initially, Li et al. developed ABab-transgenic mice with human, instead of murine, $\operatorname{TCR} \alpha \beta$ gene loci and then crossed them with

Related Article: p. 324

Conflict of interest: The authors have declared that no conflict of interest exists

Reference information: / Clin Invest. 2019;129(1):69-71. https://doi.org/10.1172/JCI125471. 
A

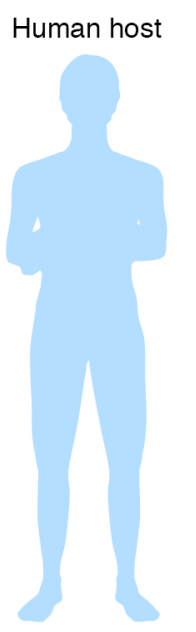

DCs

Human Human Human

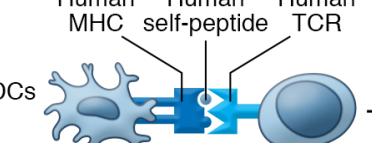

$\longrightarrow$ No positive selection Poor binding to $\mathrm{MHC}$
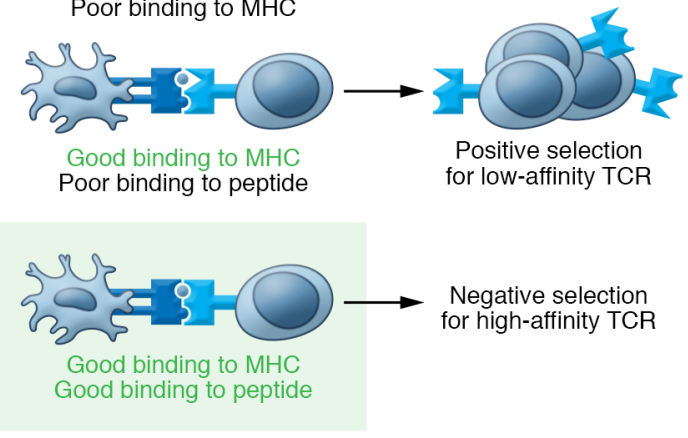

Mouse host with human TCR and $H L A$ genes
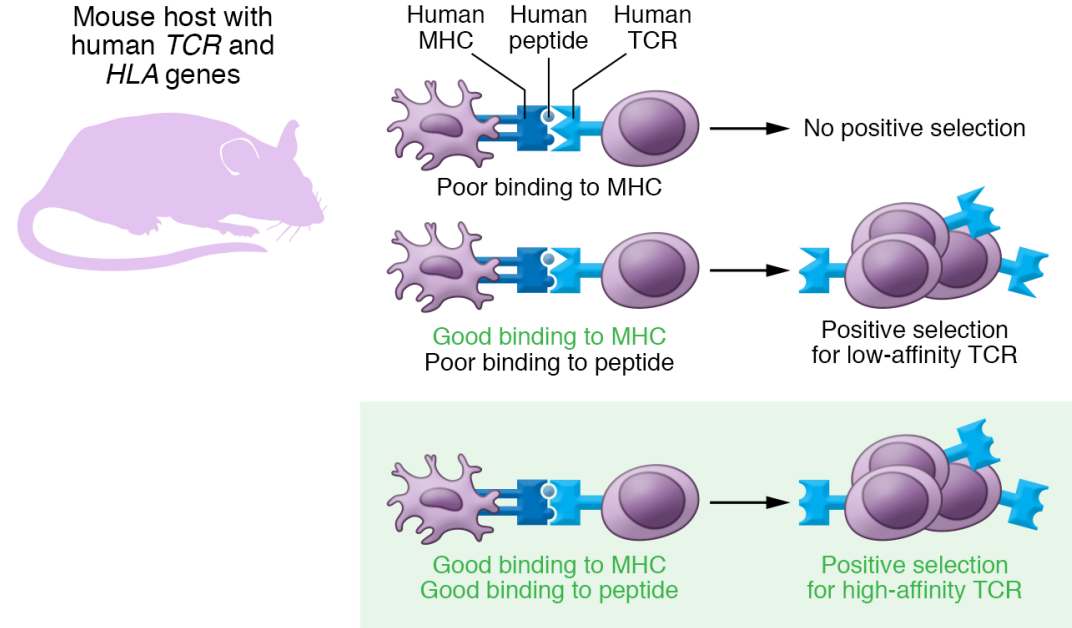

B

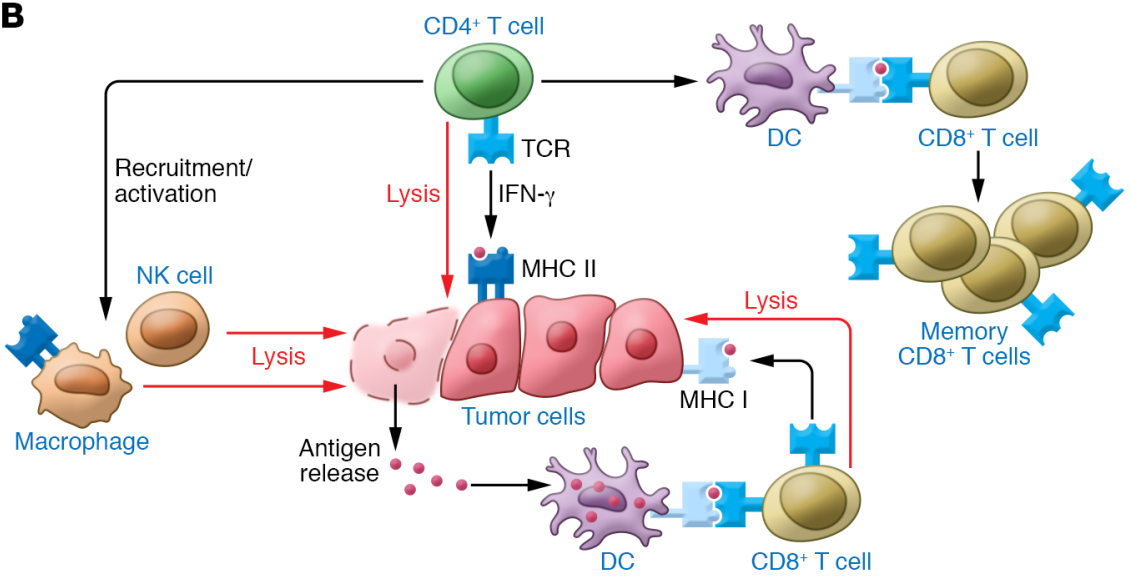

Figure 1. Optimal-affinity $C D 4^{+} \mathrm{T}$ cells for enhanced antitumor response. (A) Mouse hosts with human TCR and HLA genes generate "optimal-affinity" TCRs. As opposed to human hosts - which display self-antigens in the thymus and delete T cells that bind self-MHC and self-peptide with high affinity in a process known as negative selection - mouse hosts that lack human peptides but have human TCRs and HLA molecules can generate TCRs that bind human MHC and human peptide with high affinity. These are so-called optimal-affinity TCRs. (B) The wide range of effector and helper roles of CD4+ $\mathrm{T}$ cells in the antitumor response. CD4 ${ }^{+} \mathrm{T}$ cells can directly lyse tumor cells that have constitutive or inducible MHC II expression. Additionally, they can indirectly lead to tumor cell lysis by recruiting and activating macrophages and NK cells, which can release tumor antigens that can be presented by professional antigen-presenting cells such as dendritic cells to $\mathrm{CD}^{+} \mathrm{T}$ cells. Finally, they can also license dendritic cells, enhancing the activation and memory formation of tumor-specific CD8 ${ }^{+} \mathrm{T}$ cells.
$\mathrm{CD} 4^{+}$and $\mathrm{CD} 8^{+}$cell-based therapies may have synergistic effects, as $\mathrm{CD} 4^{+} \mathrm{T}$ cells play important helper roles in enhancing $\mathrm{CD}^{+}$activation (21), memory formation (22), and antigen spreading to nontargeted tumor epitopes $(23,24)$. In line with these important findings, this study also examined whether combining $\mathrm{CD}^{+}$and $\mathrm{CD} 8^{+} \mathrm{T}$ cells of optimalaffinity could enhance ACT efficacy.

In order to find optimal-affinity NY-ESO-1-specific $\mathrm{CD}^{+} \mathrm{T}$ cells, Poncette et al. immunized ABabDR4 mice with either NY-ESO- $1_{116-135}$ or NY-ESO-1 full-length DNA. These TCRs were shown to recognize NY-ESO ${ }_{116-135}$-loaded, NY-ESO-1-transduced, and naturally expressing NY-ESO-1 melanoma cell lines more effectively than the ones isolated from human $\mathrm{CD}^{+} \mathrm{T}$ cells. Moreover, their $\mathrm{EC}_{50}$ values in a NY-ESO-1 peptide titration assay were almost a log-fold lower than 3 of 5 human-derived TCRs $\left(10^{-10}\right.$ vs. $10^{-9} \mathrm{M}$ ), they secreted greater maximal IFN- $\gamma$ concentrations, and they had higher MFIs when stained with DR4/ NY-ESO- $1_{116-135}$ tetramer (9). One TCR, TCR-3598_2, was chosen for a combined $\mathrm{CD}^{+}$and $\mathrm{CD} 8^{+} \mathrm{ACT}$ study, as it showed no signs of alloreactivity or crossreactivity with any other naturally processed and presented human self-peptides that contain its recognition motif (9). This TCR was combined with a previously isolated HLA-A2/NY-ESO- ${ }_{157-165}$-reactive TCR (15) in an ACT fibrosarcoma model where $\mathrm{CD}^{+} \mathrm{T}$ cells could only recognize antigen on cancer cells, while the NY-ESO-1-specific $\mathrm{CD} 4^{+} \mathrm{T}$ cells could only recognize antigen cross-presented by tumor stromal cells (9). The combination of NY-ESO-1-specific CD4 ${ }^{+}$and $\mathrm{CD} 8^{+} \mathrm{T}$ cells led to tumor regression in 10 of 10 mice, although the majority of these mice did eventually develop tumors, likely due to antigen loss (9). Correspondingly, this group had the highest number of $\mathrm{CD} 8^{+} \mathrm{T}$ cells in their peripheral blood and both $\mathrm{CD}^{+}$and $\mathrm{CD} 8^{+} \mathrm{T}$ cells within their tumors. In contrast, the $\mathrm{CD} 4^{+}$-only group showed limited therapeutic benefit, suggesting that, in this system, the primary role of $\mathrm{CD}^{+} \mathrm{T}$ cells is to provide $\mathrm{T}$ cell help, rather than directly mediate antitumor activity. That said, the lack of direct $\mathrm{CD} 4^{+}$regression and the failure of the combined-treatment group 
to eradicate antigen-loss variants are possibly constraints of this engineered tumor model, rather than being indicative of true biological shortcomings of the combined $\mathrm{CD}^{+}$and $\mathrm{CD}^{+}$therapy.

\section{Concluding remarks}

There are certainly many benefits to this and other TCR gene therapy approaches for designing TCRs of optimal affinity for tumor antigens. They can provide a scalable, off-the-shelf reagent for treating many patients with a wide variety of cancers that express the corresponding tumor antigen, and engineered TCRs can be prescreened to have minimal on- or off-target toxicities. However these approaches will not confer the potential advantages associated with a polyclonal $\mathrm{T}$ cell response of a diverse spectrum of affinities and binding orientations against a single antigen that can be achieved through artificial antigenpresenting cell-based (aAPC-based) expansions (25). Moreover, as engineered TCRs are traditionally designed based on reactivity to a single dominant epitope of a tumor antigen, TCRs with reactivity to subdominant epitopes of tumor antigens remain conspicuously unexplored. Even in the case of Poncette et al., while they confirmed natural processing of NY-ESO- $1_{116-135}$ by immunizing mice with full-length NY-ESO-1 DNA, they required that all of their TCRs stain positive for DR4/NY-ESO- $1_{116-135}$ tetramer (9). In the process, however, they may have screened out TCRs that target different portions of the NY-ESO-1 protein but still have potent antitumor activity. Finally, targeting of a limited number of tumor antigens can quickly lead to outgrowth of antigen-loss variants, which may ultimately limit the value of single TCR approaches. Combined $\mathrm{CD}^{+}$and $\mathrm{CD} 4^{+}$approaches are a good start, as they can promote antigen spreading beyond targeted tumor epitopes $(23,24)$. As the importance of clinically relevant ACT therapies expands, further mechanistic studies comparing the potential clinical benefit of polyclonal antitumor responses over single high-affinity TCR approaches as well as more sophisticated systems for generating diverse TCR repertoires will ultimately help us develop and optimize ACT therapies.

\section{Acknowledgments}

This work was funded in part through the National Science Foundation (to AI), NIH/NCI Cancer Center Support Grant P01-AI072677 (to JPS), R01-CA108835 (to JPS), R21-CA185819 (to JPS), R21 EB023411, TEDCO/Maryland Innovation Initiative, and the Wallace H. Coulter Foundation (to JPS).

Address correspondence to: Jonathan Schneck, Department of Pathology, Johns Hopkins University School of Medicine, 733 N. Broadway, Miller Research Building Room 639, Baltimore, Maryland 21205, USA. Phone: 410.614.4589; Email: jschnec1@jhmi.edu.

1. Dembić Z, et al. Transfer of specificity by murine alpha and beta T-cell receptor genes. Nature. 1986;320(6059):232-238.

2. Morgan RA, et al. Cancer regression in patients after transfer of genetically engineered lymphocytes. Science. 2006;314(5796):126-129.

3. Johnson LA, et al. Gene therapy with human and mouse $\mathrm{T}$-cell receptors mediates cancer regression and targets normal tissues expressing cognate antigen. Blood. 2009;114(3):535-546.

4. Rosati SF, et al. A novel murine T-cell receptor targeting NY-ESO-1. J Immunother. 2014;37(3):135-146.

5. Sadovnikova E, Jopling LA, Soo KS, Stauss HJ. Generation of human tumor-reactive cytotoxic T cells against peptides presented by non-self HLA class I molecules. Eur J Immunol. 1998;28(1):193-200.

6. Robbins PF, et al. Single and dual amino acid substitutions in TCR CDRs can enhance antigen-specific $\mathrm{T}$ cell functions. J Immunol. 2008;180(9):6116-6131.

7. Holler PD, Chlewicki LK, Kranz DM. TCRs with high affinity for foreign $\mathrm{pMHC}$ show self-reactivity. Nat Immunol. 2003;4(1):55-62.

8. Li Y, et al. Directed evolution of human T-cell receptors with picomolar affinities by phage display. Nat Biotechnol. 2005;23(3):349-354.

9. Poncette L, Chen X, Lorenz FKM, Blankenstein T. Effective NY-ESO-1-specific MHC II-restricted $\mathrm{T}$ cell receptors from antigen-negative hosts enhance tumor regression. J Clin Invest. 2019;129(1):324-335.

10. Li LP, et al. Transgenic mice with a diverse human T cell antigen receptor repertoire. Nat Med. 2010;16(9):1029-1034.

11. Davis JL, Theoret MR, Zheng Z, Lamers CH, Rosenberg SA, Morgan RA. Development of human anti-murine $\mathrm{T}$-cell receptor antibodies in both responding and nonresponding patients enrolled in TCR gene therapy trials. Clin Cancer Res. 2010;16(23):5852-5861.

12. Morgan RA, et al. Cancer regression and neurological toxicity following anti-MAGE-A3 TCR gene therapy. J Immunother. 2013;36(2):133-151.

13. Scott-Browne JP, White J, Kappler JW, Gapin L, Marrack P. Germline-encoded amino acids in the alphabeta T-cell receptor control thymic selection. Nature. 2009;458(7241):1043-1046.

14. Chen X, Poncette L, Blankenstein T. Human TCR$\mathrm{MHC}$ coevolution after divergence from mice includes increased nontemplate-encoded CDR3 diversity. JExp Med. 2017;214(11):3417-3433.

15. Obenaus $\mathrm{M}$, et al. Identification of human T-cell receptors with optimal affinity to cancer antigens using antigen-negative humanized mice. Nat Biotechnol. 2015;33(4):402-407.

16. Kreiter S, et al. Mutant MHC class II epitopes drive therapeutic immune responses to cancer. Nature. 2016;520(7549):692-696.

17. Sahin U, et al. Personalized RNA mutanome vaccines mobilize poly-specific therapeutic immunity against cancer. Nature. 2017;547(7662):222-226.

18. Quezada SA, et al. Tumor-reactive CD4(+) T cells develop cytotoxic activity and eradicate large established melanoma after transfer into lymphopenic hosts. J Exp Med. 2010;207(3):637-650.

19. Perez-Diez A, et al. CD4 cells can be more efficient at tumor rejection than CD8 cells. Blood. 2007;109(12):5346-5354.

20. Hunder NN, et al. Treatment of metastatic melanoma with autologous $\mathrm{CD} 4^{+} \mathrm{T}$ cells against NY-ESO-1. NEngl JMed. 2008;358(25):2698-2703.

21. Antony $\mathrm{PA}$, et al. $\mathrm{CD} 8^{+} \mathrm{T}$ cell immunity against a tumor/self-antigen is augmented by $\mathrm{CD} 4^{+} \mathrm{T}$ helper cells and hindered by naturally occurring $\mathrm{T}$ regulatory cells. J Immunol. 2005;174(5):2591-2601.

22. Bourgeois C, Tanchot C. Mini-review CD4 T cells are required for CD8 $\mathrm{T}$ cell memory generation. Eur J Immunol. 2003;33(12):3225-3231.

23. Schietinger A, Philip M, Liu RB, Schreiber $\mathrm{K}$, Schreiber H. Bystander killing of cancer requires the cooperation of $\mathrm{CD} 4(+)$ and CD8(+) $\mathrm{T}$ cells during the effector phase. J Exp Med. 2010;207(11):2469-2477.

24. $\mathrm{Li} \mathrm{K}$, et al. Adoptive cell therapy with $\mathrm{CD}^{+} \mathrm{T}$ helper 1 cells and CD8+ cytotoxic T cells enhances complete rejection of an established tumour, leading to generation of endogenous memory responses to non-targeted tumour epitopes. Clin Transl Immunology. 2017;6(10):e160

25. Perica K, et al. Enrichment and expansion with nanoscale artificial antigen presenting cells for adoptive immunotherapy. ACS Nano. 2015;9(7):6861-6871. 